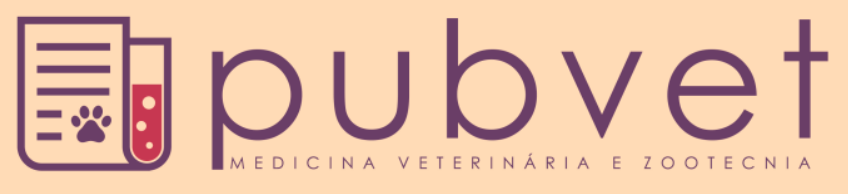

https://doi.org/10.22256/pubvet.v12n5a83.1-8

\title{
Percepção dos estudantes sobre modelo de baixo custo para treinamento de ovariosalpingohisterectomia em pequenos animais
}

\author{
Natália Noreika Kano ${ }^{1}$, Flávia Carolina Meira Collere ${ }^{2}$, Letícia Fernanda \\ Laube ${ }^{\bullet}$, Simone Tostes de Oliveira Stedile ${ }^{\bullet}$, Roberta Carareto ${ }^{\ominus *}$ \\ ${ }^{l}$ Acadêmica do curso Medicina Veterinária da Universidade Federal do Paraná (UFPR), Curitiba -PR Brasil. E-mail: \\ nataliakano11@gmail.com ${ }^{2}$ Acadêmica do curso Medicina Veterinária, UFPR, Curitiba -PR Brasil. E-mail: flaviacollere@gmail.com; \\ ${ }^{3}$ Acadêmica do curso Medicina Veterinária, UFPR, Curitiba-PR Brasil. E-mail: leticia_laube@hotmail.com; ${ }^{4}$ Professora do Departamento \\ de Medicina Veterinária, UFPR. Curitiba -PR Brasil. E-mail: tostesimone@gmail.com; ${ }^{5}$ Professora do Departamento de Medicina \\ Veterinária, UFPR. Curitiba-PR Brasil. E-mail: robertacarareto@hotmail.com \\ *Autor para correspondência
}

RESUMO. Na Medicina Veterinária o uso de métodos alternativos tem sido explorado com intuito de substituir a utilização de animais como recurso didático. $\mathrm{O}$ objetivo deste estudo foi analisar a opinião dos estudantes em relação ao uso de um modelo de baixo custo para treinamento de ovariosalpingohisterectomia em pequenos animais antes da realização no animal in vivo. Foram envolvidos 21 estudantes da graduação da disciplina de Técnica Cirúrgica Veterinária, que realizaram o treinamento no modelo, antes de realizar a cirurgia no animal e depois responderam um questionário a respeito do uso do modelo, incluindo organização da equipe, segurança emocional e treinamento da técnica. A maioria dos estudantes $(95,2 \%)$ consideraram que o treinamento melhorou o fluxo de tarefas, $85,7 \%$ sentiram-se mais seguros em realizar a cirurgia no animal vivo após o treinamento com o modelo e 76,2\% consideraram que o treinamento acarretou melhora na habilidade cirúrgica para realização da cirurgia. Também houve redução em 23,2\% (32 minutos) no tempo cirúrgico no animal vivo, comparado à turma do ano anterior sem treinamento no modelo. A utilização do modelo teve resultados positivos, pois além de ser bem aceita pelos estudantes, trouxe maior confiança e habilidade técnica durante a cirurgia, diminuindo o tempo de procedimento.

Palavras chave: cão, cirurgia veterinária, ensino, gato, métodos alternativos

\section{Perception of students about a low cost model for training ovariosalpingohisterectomy in small animals}

\begin{abstract}
In Veterinary Education, non-harmful alternatives methods have been explored in order to replace the use of animals as a teaching resource. The objective of this study was to analyze the students' opinion regarding the use of a low cost model for training ovariaosalpingohisterectomy in small animals before in vivo performance. There were 21 veterinary medicine students from a Veterinary Surgical Teaching class enrolled in the study. They underwent training in the model before performing the surgery on the animal and then answered a questionnaire regarding the use of the model, including team organization, emotional safety and technique training. Among the students, 95.2\% considered that the training improved the flow of tasks, $85.7 \%$ felt safer in performing the surgery on the live animal after training with the model and $76.2 \%$ considered that the training resulted in an improvement in the surgical ability to perform the surgery. There was also a $23.2 \%$ decrease ( 32 minutes) in surgical time in the live animal, compared to the previous year's group without training in the model. The use of the model had positive
\end{abstract}


results, because besides being well accepted by the students, it brought greater confidence and technical skill during the surgery, reducing the time of the procedure.

Keywords: alternative methods, cat, dog, teaching, veterinary surgery

\title{
Percepción de los estudiantes sobre modelo de bajo costo para entrenamiento de ovariosalpingohisterectomía en pequeños animales
}

\begin{abstract}
RESUMEN. En la Medicina Veterinaria el uso de métodos alternativos se ha explorado con el propósito de sustituir la utilización de animales como recurso didáctico. El objetivo de este estudio fue analizar la opinión de los estudiantes en relación al uso de un modelo de bajo costo para entrenamiento de ovariosalpingohisterectomía en pequeños animales antes de la realización en el animal in vivo. Fueron involucrados 21 estudiantes de la graduación de la disciplina de Técnica Cirúrgica Veterinaria, que realizaron el entrenamiento en el modelo, antes de realizar la cirugía en el animal y luego respondieron un cuestionario acerca del uso del modelo, incluyendo la organización del equipo, la seguridad emocional y el entrenamiento de la técnica. La mayoría de los estudiantes $(95,2 \%)$ consideró que el entrenamiento mejoró el flujo de tareas, $85,7 \%$ se sintieron más seguros en realizar la cirugía en el animal vivo después del entrenamiento con el modelo y $76,2 \%$ consideraron que el entrenamiento acarreó una mejora en la habilidad cirúrgica para la realización de la cirugía. También hubo reducción en 23,2\% (32 minutos) en el tiempo cirúrgico en el animal vivo, comparado a la clase del año anterior, sin entrenamiento en el modelo. La utilización del modelo ha tenído resultados positivos, pues además de ser bien aceptada por los estudiantes, trajo mayor confianza y habilidad técnica durante la cirugía, disminuyendo el tiempo de procedimiento.
\end{abstract}

Palabras clave: perro, cirugía veterinaria, enseñanza, gato, métodos alternativos

\section{Introdução}

A utilização de animais vivos com fins didáticos para aulas práticas em cirurgia veterinária tem sido discutida no âmbito acadêmico, principalmente no que se refere ao bem-estar animal, levando em consideração o conceito dos 3 "R's" (Reduction, Refinement e Replacement) (Knight, 2007). O aprendizado do estudante pode ser afetado ao submete-lô à pressão e medo, devido a falta de treinamento prévio (Griffon et al., 2000), o que pode acarretar um aumento do tempo de procedimento cirúrgico. Por esses motivos, métodos alternativos estão sendo desenvolvidos, entre eles, modelos sintéticos (Griffon et al., 2000, Smeak, 2007), treinamentos por vídeo (Knight, 2008, Roshier et al., 2011), simulação computadorizada (Knight, 2008) e utilização de cadáveres conservados (Silva et al., 2007, Souza \& Matera, 2015). Os benefícios ocorrem tanto em prol dos animais, como dos estudantes, pois permite realizar o procedimento com maior tranquilidade, sanar dúvidas e aperfeiçoar a técnica. Estudos mostraram que métodos alternativos podem proporcionar aos estudantes aprendizado similar, ou até mesmo superior, quando comparado ao uso do animal vivo (Holmberg et al., 1993, Kosachenco et al., 2007, Silva et al., 2007, Tefera, 2011). A ovariosalpingohisterectomia (OSH) em pequenos animais é a cirurgia eletiva mais realizada na rotina (Howe, 2006, Quessada et al., 2009). É uma técnica empregada com frequência nas aulas práticas de técnica cirúrgica veterinária, pois além de promover benefícios ao animal, auxilia no controle populacional (Bojrab, 2005, Johnston et al., 2001, Slatter, 2007, Stone, 2007). A técnica de OSH envolve os três tempos cirúrgicos (diérese, hemostasia e síntese) e requer bom conhecimento anatômico. Desta forma, o objetivo deste trabalho foi descrever a percepção dos estudantes quanto ao uso de um modelo sintético como treinamento prévio à cirurgia de OSH em cadelas e gatas.

\section{Material e Métodos}

Foi realizado um treinamento com os estudantes de Medicina Veterinária que mimetizasse desde a paramentação dos mesmos até a conclusão do procedimento cirúrgico, utilizando um modelo sintético com representação da anatomia do aparelho geniturinário de cadelas e gatas, para simulação da técnica de OSH. Para tal, os estudantes do $3^{\circ}$ ano de Medicina 
Veterinária, cursando a disciplina de Técnica Cirúrgica Veterinária foram instruídos a confeccionar, em duplas, seu próprio modelo, para posterior utilização em aula. Os estudantes deveriam considerar a anatomia do sistema geniturinário, com ênfase na localização dos ovários em relação aos rins e dos cornos e corpo uterino em relação à vesícula urinária. $\mathrm{O}$ modelo foi confeccionado a partir de materiais de baixo custo como garrafas pet, espumas e balões de látex (Figura 1), baseado no modelo de Silveira et al. (2015).

A aula prática ocorreu no Bloco Cirúrgico de Pequenos Animais, mesmo local utilizado para as aulas práticas com animais vivos. A aula envolveu as principais etapas de um procedimento cirúrgico, incluindo antissepsia de mãos e braços, paramentação, organização da mesa de instrumentais, colocação de campos cirúrgicos e técnica cirúrgica. Ao entrar no centro cirúrgico, os estudantes posicionaram os modelos nas mesas cirúrgicas, mimetizando animais vivos. $\mathrm{Na}$ sequência, treinaram o modo correto de abrir embalagens e materiais estéreis e foram para a área de assepsia, na qual praticaram a correta escovação de unhas, dedos, mãos e braços com escovas-esponjas estéreis de clorexidina, seguida da secagem de mãos. A paramentação foi realizada na sala cirúrgica com a colocação dos aventais e luvas estéreis. Após a paramentação, cada equipe recebeu uma caixa estéril com materiais cirúrgicos para OSH e montaram a mesa de instrumentais. Foi realizada a antissepsia do modelo, com passagens sucessivas de solução de álcool $70 \%$ e Polivinilpirrolidona-iodo (PVPI), e então foram colocados os campos cirúrgicos estéreis sobre o modelo sintético (Figura 2A), fixando-o com as Pinças de Backaus, marcando o início do procedimento cirúrgico (Figura 2B).

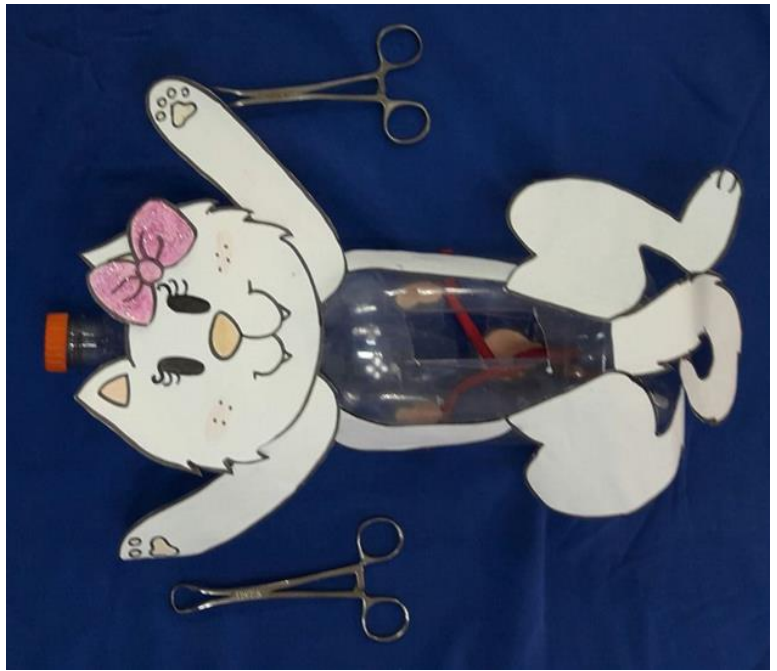

Figura 1. Modelo para treinamento de OSH em pequenos animais, simulando o sistema geniturinário. As bordas de uma garrafa pet foram cortadas e por dentro foram colocados materiais como balão ou figuras de papel para simular os rins e vesícula urinária. Para os ovários e os cornos uterinos foram utilizados balões de látex tipo bola e tipo canudo, respectivamente. Os dois balões que representavam os cornos foram unidos em sua porção caudal, formando a cérvix.
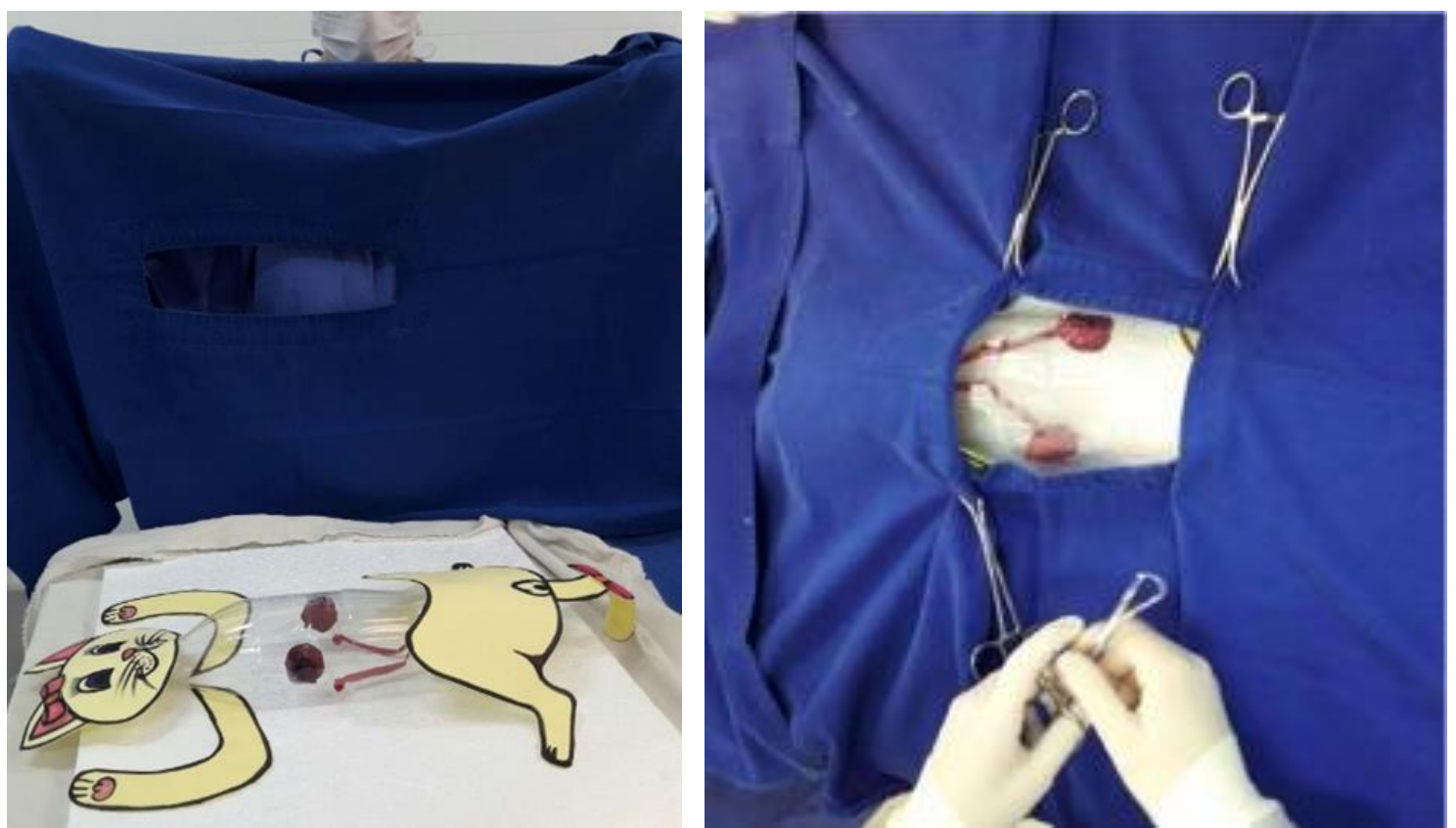

Figura 2. A. Preparação do modelo sintético para simulação de OSH com a colocação do campo cirúrgico estéril. B. Colocação das pinças de Backaus. 
A técnica de OSH das três pinças foi realizada como descrita por MacPhail (2014). Foi realizada uma incisão na garrafa pet com bisturi (Figura 3A), e ampliada com o auxílio de uma tesoura, para possibilitar a manipulação dos órgãos. $\mathrm{O}$ ovário foi localizado a partir da identificação do rim, e, através do rompimento digital do ligamento suspensório ovariano, permitiu-se sua exteriorização (Figura 3B).

Foram colocadas três pinças hemostáticas no pedículo ovariano (Figura 4A), e fez-se a incisão do pedículo. Em seguida, foi simulada a ligadura dos vasos, a partir das bexigas de látex, com fio absorvível sintético poliglactina 2-0 (Figura 4B).
A pinça hemostática mais distal ao ovário foi então removida e os estudantes foram alertados sobre possíveis hemorragias que ocorrem nesta etapa no animal vivo, quando a ligadura dos vasos não é eficiente, uma vez que o modelo não simulou sangue. $\mathrm{O}$ mesmo procedimento foi realizado no ovário contralateral e corno uterino. A partir de tração cranial do útero, a cérvix foi exposta, foram colocadas as pinças hemostáticas acima da cérvix e o corpo uterino foi incisado e ligado. $\mathrm{O}$ modelo não possibilita a síntese, porém os estudantes foram instruídos sobre as etapas de uma laparorrafia.

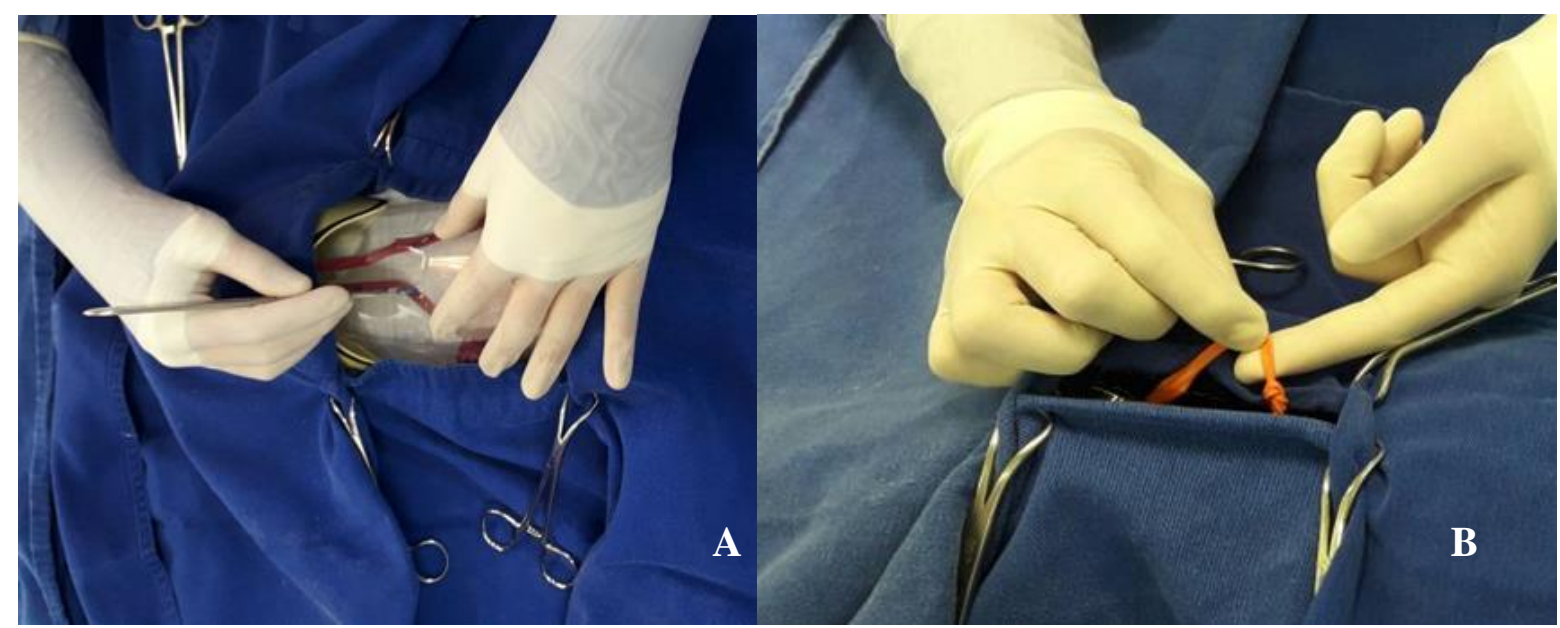

Figura 3. A. Incisão da garrafa pet, simulando o acesso cirúrgico para OSH. B. Exteriorização do ovário e corno uterino.
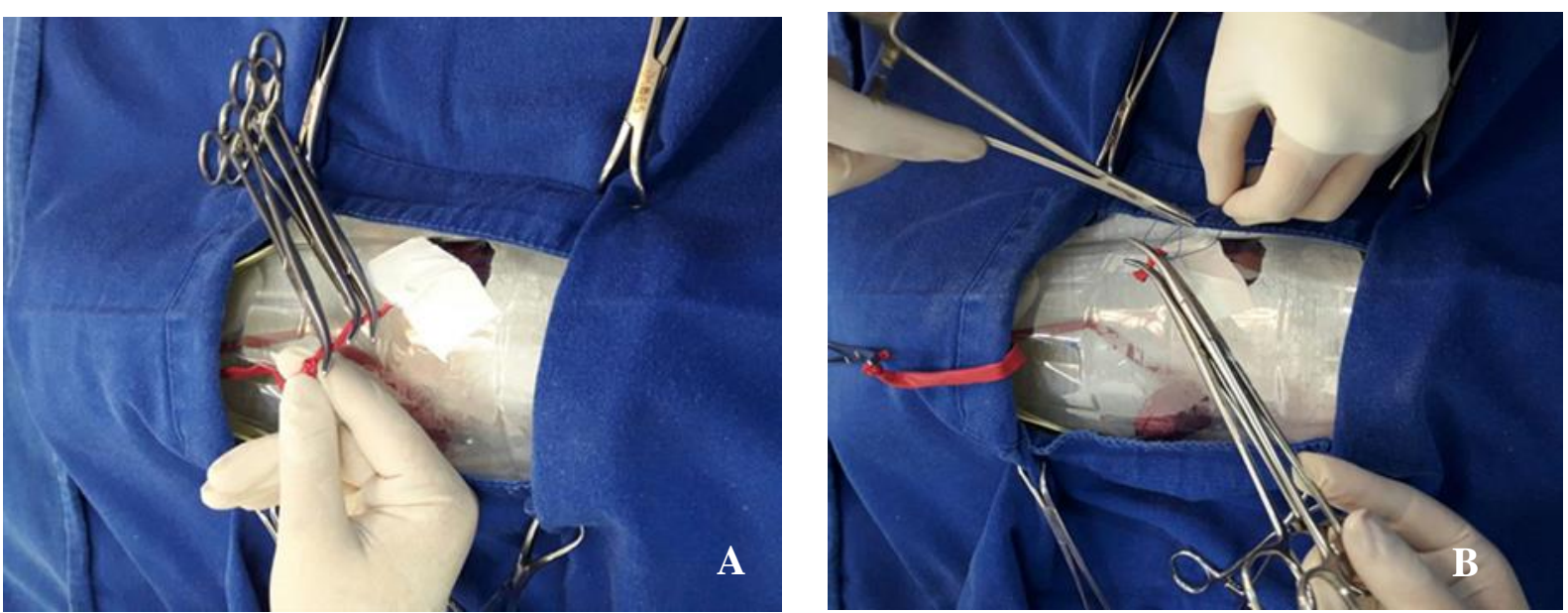

Figura 4. A. Simulação de OSH pela técnica das três pinças no modelo sintético com a colocação das três pinças hemostáticas no pedículo. B. Ressecção e ligadura dos vasos ovarianos.

Após as aulas práticas com os modelos, em uma segunda etapa, durante o ano letivo, os estudantes realizaram cirurgias eletivas de $\mathrm{OSH}$ em cadelas e gatas com idade entre 8 meses e 6 anos de idade. O tempo cirúgico no animal vivo, correspondendo desde a incisão até o último ponto de pele, foi anotado, para posterior comparação com a turma do ano anterior, que não fez o uso do modelo. Para avaliação do modelo, foram aplicados questionários anônimos aos estudantes que participaram do treinamento prévio e atuaram como cirurgiões nas OSHs em animais vivos. Os estudantes opinaram sobre a utilização do modelo como treinamento de OSH, desde a paramentação até a finalização da cirurgia. 
O questionário se referiu à percepção dos estudantes quanto ao aproveitamento do uso do modelo como treinamento na aula prática, qualidade do modelo, organização das equipes e fluxo de tarefas, segurança emocional para realização da técnica in vivo após treinamento prévio e desenvolvimento das habilidades técnicas dos estudantes. O tempo cirúrgico foi comparado pelo teste $\mathrm{t}$ de Student, sendo utilizadointervalo de confiança de 95\% (Software Excel, Microsoft Office Professional Plus 2013).

\section{Resultados e Discussão}

No total, 21 estudantes participaram da pesquisa. Após o treinamento com o modelo e a cirurgia com o animal vivo, os estudantes opinaram sobre a utilidade do modelo como treinamento prévio de OSH. A avaliação dos estudantes sobre a aula prática realizada com o modelo encontra-se na Tabela 1. Nenhum estudante concordou que a simulação com o modelo neste formato de aula, abrangendo desde a paramentação até o fim da cirurgia, tenha sido uma perda de tempo. A maioria dos estudantes $(95,2 \%)$ afirmou que a realização do treinamento e simulação melhorou o fluxo de tarefas dos integrantes da equipe cirúrgica, por permitir conhecimento prévio do local e das tarefas a serem realizadas. No âmbito cirúrgico, o processo cirúrgico como um todo é otimizado quando as responsabilidades $\mathrm{e}$ as funções de todos os membros da equipe cirúrgica são definidas antecipadamente (Caplan, 2014).

Tabela 1. Opinião dos estudantes $(n=21)$ quanto à aula prática com modelo, levando em consideração a simulação da cirurgia de OSH desde a paramentação e a formação da equipe cirúrgica, até o fim do procedimento.DF - Discordo fortemente; $\mathrm{D}$ Discordo; $\mathrm{N}$ - Neutro; $\mathrm{C}$ - Concordo; CF Concordo fortemente.

\begin{tabular}{lccccc}
\hline Questões & $\mathrm{DF}$ & $\mathrm{D}$ & $\mathrm{N}$ & $\mathrm{C}$ & $\mathrm{CF}$ \\
\hline $\begin{array}{l}\text { Foi uma perda de tempo, deveria ter sido } \\
\text { apenas com animal vivo }\end{array}$ & 16 & 4 & 1 & 0 & 0 \\
$\begin{array}{l}\text { Melhorou o fluxo de tarefas de cada } \\
\text { integrante da equipe cirúrgica }\end{array}$ & 0 & $(19 \%)$ & $(4,8 \%)$ & $(0 \%)$ & $(0 \%)$ \\
\hline
\end{tabular}

Sobre a segurança ao executar o procedimento no animal vivo após a realização do treinamento, a maioria dos estudantes disse se sentir mais segura e também que o treinamento melhorou suas habilidades técnicas para a realização da cirurgia (Tabela 2). Uma adequada consolidação de competências psicomotoras essenciais para a realização da técnica in vivo pode ser obtida a partir de um treinamento aliado à ausência de estresse e medo, sem a utilização de animais vivos logo na primeira aula (Griffon et al., 2000, Tudury \& Potier, 2009, Silveira et al., 2015). Nesse caso, o uso do modelo aumentou a confiança e habilidade dos estudantes durante a cirurgia nos animais, pois durante o treinamento a discussão das etapas/tempos cirúrgicos se faz de forma mais tranquila, sem preocupação de causar danos ao animal, como ocorreria na cirurgia real. Em um estudo utilizando modelo de OSH em gatas como treinamento, verificou-se que se o ensino tivesse ocorrido diretamente no animal vivo, teria provocado um impacto emocional muito negativo em alguns estudantes (Badman et al., 2016).

Tabela 2. Opinião dos estudantes $(n=21)$ sobre a importância do treinamento com o modelo de OSH antes da cirurgia no animal vivo, em relação à sua segurança em realizar o procedimento e em relação ao aprendizado com o modelo de OSH. DF - Discordo fortemente; D - Discordo; N - Neutro; C - Concordo; CF Concordo fortemente.

\begin{tabular}{lccccc}
\hline Questões & DF & D & N & C & CF \\
\hline $\begin{array}{l}\text { Eu me senti mais seguro para realizar a } \\
\text { cirurgia }\end{array}$ & 1 & 0 & 2 & 12 & 6 \\
Melhorou minhas habilidades técnicas & 0 & $(0 \%)$ & $(9,5 \%)$ & $(57,1 \%)$ & $(28,6 \%)$ \\
para a realização da cirurgia & $(0 \%)$ & $(0 \%)$ & $(23,8 \%)$ & $(47,6 \%)$ & 6 \\
$\begin{array}{l}\text { Relembrei a anatomia dos órgãos a serem } \\
\text { manipulados }\end{array}$ & 3 & 0 & 1 & 7 & 10 \\
\hline
\end{tabular}


A maioria dos estudantes se mostrou satisfeita com o uso do modelo, considerando-o necessário como forma de treinamento prévio, e considerando-o útil em auxiliar a superar a dificuldade da prática no animal vivo. Entretanto, a maioria afirmou que o modelo não permitiu a visualização de erros e acidentes transcirúrgicos (Tabela 3). Quando questionados sobre a substituição das aulas práticas com animais vivos apenas pelo uso de modelos, 95,2\% dos estudantes discorda (Tabela 3). Quanto à preferência na sequência de treinamento para seu aprendizado, $76,2 \%$ dos estudantes gostariam de treinar primeiramente em modelos e após, em cadáver, para então realizar a cirurgia em um animal vivo (Tabela 4). Em estudo conduzido por Griffon et al. (2000) compararam-se o uso de cadáver com o uso de modelo, prévio a OSH em animal vivo, e concluiu-se que o modelo foi mais efetivo para aprendizado de habilidade cirúrgica básica e maior familiaridade com a técnica, bem como para observação de possíveis hemorragias no caso de técnica incorreta da ligadura, uma vez que tal modelo era acoplado a uma bomba peristáltica para simular sangramento. No presente trabalho, mesmo que o cadáver estivesse disponível como etapa de treinamento, como sugerido pelos estudantes, a deficiência em se produzir uma hemorragia, no caso da técnica incorreta da ligadura, permaneceria.

Os estudantes citaram como pontos fortes do modelo principalmente o treinamento da técnica das três pinças e da ligadura, propiciando maior segurança no momento de realizar os mesmos passos no animal vivo. Outro ponto citado como forte foi a prática da cirurgia sem colocar a vida de um animal em risco, permitindo maior tranquilidade ao realizar a técnica posteriormente. Por tratar-se de um modelo simples, de baixo custo, fácil e rápida confecção, a possibilidade de treinar quantas vezes se julgar necessário torna-se viável, aumentando as habilidades do estudante. $\mathrm{O}$ treinamento por meio de modelos, ao não colocar o bem-estar e a vida do paciente em risco, permite repetições até aperfeiçoamento da técnica (Holmberg et al., 1993, Smeak et al., 1994, Tefera, 2011). Ao comparar um modelo de silicone (\$677) com um modelo mais simples, de pano e espuma (\$64), verificou-se que o modelo mais simples/ de baixo custo também foi efetivo, quando comparado ao modelo de alto custo (Read et al., $\underline{2016) \text {. }}$

Tabela 3. Opinião dos estudantes $(n=21)$ sobre a utilização do modelo para treinamento de OSH nas aulas de Técnica Cirúrgica Veterinária. (DF - Discordo fortemente; D - Discordo; N - Neutro; C - Concordo; CF Concordo fortemente)

\begin{tabular}{|c|c|c|c|c|c|}
\hline Questões & DF & $\mathrm{D}$ & $\mathrm{N}$ & $\mathrm{C}$ & $\mathrm{CF}$ \\
\hline $\begin{array}{l}\text { As aulas práticas com animais vivos } \\
\text { poderiam ser substituídas apenas pelo uso } \\
\text { de modelos }\end{array}$ & $13(61,9 \%)$ & $\begin{array}{c}7 \\
(33,3 \%)\end{array}$ & $\begin{array}{c}1 \\
(4,8 \%)\end{array}$ & $\begin{array}{c}0 \\
(0 \%)\end{array}$ & $\begin{array}{c}0 \\
(0 \%)\end{array}$ \\
\hline $\begin{array}{l}\text { Os modelos permitem a visualização de } \\
\text { erros e acidentes trans cirúrgicos }\end{array}$ & $\begin{array}{c}4 \\
(19 \%)\end{array}$ & $\begin{array}{c}8 \\
(38,1 \%)\end{array}$ & $\begin{array}{c}1 \\
(4,8 \%)\end{array}$ & $\begin{array}{c}6 \\
(28,6 \%)\end{array}$ & $\begin{array}{c}2 \\
(9,5 \%)\end{array}$ \\
\hline $\begin{array}{l}\text { Não achei necessário usar o modelo antes } \\
\text { como forma de treinamento }\end{array}$ & $\begin{array}{c}11 \\
(52,3 \%)\end{array}$ & $\begin{array}{c}9 \\
(42,9 \%)\end{array}$ & $\begin{array}{c}1 \\
(4,8 \%)\end{array}$ & $\begin{array}{c}0 \\
(0 \%)\end{array}$ & $\begin{array}{c}0 \\
(0 \%)\end{array}$ \\
\hline $\begin{array}{l}\text { O modelo foi útil em auxiliar a superar a } \\
\text { dificuldade no animal em vivo }\end{array}$ & $\begin{array}{c}1 \\
(4,8 \%)\end{array}$ & $\begin{array}{c}4 \\
(19 \%)\end{array}$ & $\begin{array}{c}3 \\
(14,3 \%)\end{array}$ & $\begin{array}{c}10 \\
(47,6 \%)\end{array}$ & $3(14,3 \%)$ \\
\hline
\end{tabular}

Tabela 4. Opinião dos estudantes $(n=21)$ sobre suas preferências da sequência de treinamento da técnica de OSH na disciplina de Técnica Cirúrgica Veterinária.

\begin{tabular}{lc}
\hline Sequencia de treinamento & Número de estudantes $(\%)$ \\
\hline Modelo, cadáver, animal vivo & $16(76,2 \%)$ \\
Cadaver, vivo & $3(14,3 \%)$ \\
Modelo, vivo & $2(9,5 \%)$ \\
Apenas animal vivo & $0(0 \%)$ \\
Apenas cadáver & $0(0 \%)$ \\
Apenas modelo & $0(0 \%)$ \\
\hline
\end{tabular}


Quanto às dificuldades enfrentadas no treinamento com modelo, $61,9 \%$ dos estudantes argumentaram que a maior dificuldade foi em realizar a incisão, enquanto no animal vivo, $38 \%$ dos estudantes relataram dificuldade na localização dos ovários e útero durante a OSH. Esses dados estão relacionados com os respostas apresentadas pelos estudantes, como pontos positivos e negativos em relação ao modelo. A dificuldade dos estudantes em realizar a incisão no modelo decorreu devido ao material de baixo custo utilizado não ser fiel à textura da pele dos animais. Outro ponto fraco apresentado incluiu a falta de estruturas que mimetizassem os demais órgãos da cavidade abdominal, bem como a ausência de vasos sanguíneos que simulassem sangramento, como ocorria no animal vivo. Mesmo assim, a utilização do treinamento com modelo para OSH despertou grande interesse dos estudantes, sendo que $61,9 \%$ afirmaram interesse em seguir a área de cirurgia de pequenos animais.

O tempo médio gasto na cirurgia de OSH em cadelas vivas, no ano anterior, por estudantes que não realizaram nenhum tipo de treinamento prévio, foi de $137,9( \pm 22,7)$ minutos, enquanto que no ano do presente trabalho, o tempo médio foi de 105,9 $( \pm 13,2)$ minutos (Figura 5). Quando comparados os tempos cirúrgicos médios, houve uma redução de 32 minutos $(23,2 \%$ a menos que no ano anterior), apresentando diferença significativa $(\mathrm{P}<0,001)$.Desta forma, foi possível verificar que o treinamento prévio com o modelo trouxe maior agilidade aos estudantes durante a cirurgia.

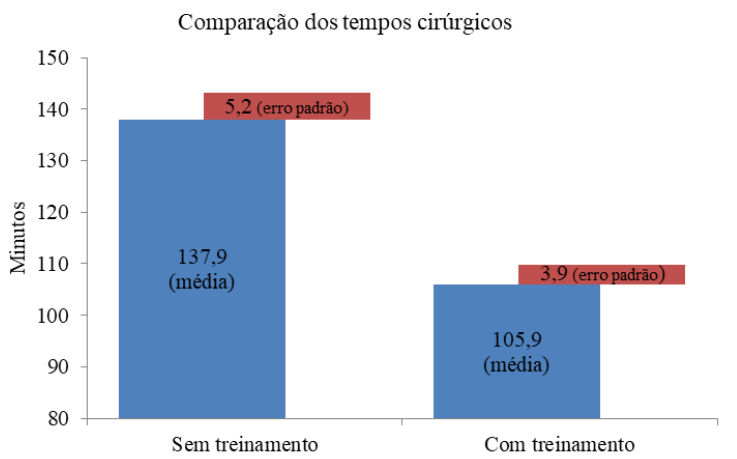

Figura 5. Tempo médio gasto para realizar OSH eletiva em cadelas, por estudantes sem ou com treinamento prévio com modelo, na disciplina de Técnica Cirúrgica Veterinária.

A utilização de métodos de ensino variados para treinamento e realização de técnicas cirúrgicas contribui para o desenvolvimento da ética profissional, além do aprendizado dos estudantes, garantindo a preservação da saúde e bem-estar animal (Silveira et al., 2015), sendo uma tendência a busca por tais métodos (Griffon et al., 2000). Sugere-se para futuras pesquisas do uso de modelo de baixo custo, como complemento à prática de $\mathrm{OSH}$ em animal vivo, melhorias no modelo em relação à presença de outras estruturas simulando os órgãos da cavidade abdominal, como intestinos, baço, vesícula urinária. A confecção de um sistema que permita simular com maior precisão a técnica de ligadura e possíveis hemorragias também deve ser levada em consideração.

\section{Conclusão}

A utilização do modelo sintético para treinamento de OSH foi bem aceita pelos estudantes, possibilitou o desenvolvimento de habilidade cirúrgica e aumentou a segurança emocional. O treinamento prévio no modelo reduziu o tempo cirúrgico de OSH em cadelas, quando comparado ao ano anterior, no qual os alunos não tiveram treinamento prévio com modelos.

\section{Referências |Bibliográficas}

Badman, M., Tullberg, M., Höglund, O. V. \& Hagman, R. 2016. Veterinary Student Confidence after Practicing with a New Surgical Training Model for Feline Ovariohysterectomy. Journal of Veterinary Medical Education, 43, 427-433.

Bojrab, M. J. 2005. Técnicas atuais em cirurgia de pequenos animais. Editora Roca, São Paulo.

Caplan, E. R. 2014. Instalações cirúrgicas, equipamentos, pessoal e cuidados e manutenção do ambiente cirúrgico. In: Fossum, T. W. (ed.) Cirurgia de pequenos animais. Elsevier, Rio de Janeiro, Brasil.

Griffon, D. J., Cronin, P., Kirby, B. \& Cottrell, D. F. 2000. Evaluation of a hemostasis model for teaching ovariohysterectomy in veterinary surgery. Veterinary Surgery, 29, 309-316.

Holmberg, D. L., Cockshutt, J. R. \& Basher, A. W. P. 1993. Use of a dog abdominal surrogate for teaching surgery. Journal Veterinary Medical Education, 20, 61-62.

Howe, L. M. 2006. Surgical methods of contraception and sterilization. Theriogenology, 66, 500-509.

Johnston, S. D., Olson, M. V., Johnston, P. S. S. D., Kustritz, M. V. R. \& Olson, P. N. S. 2001. Canine and feline theriogenology. WB Saunders, Philapdelphia. 
Knight, A. 2007. The effectiveness of humane teaching methods in veterinary education. Altex, 24, 91-109.

Knight, A. 2008. Non-animal methodologies within biomedical research and toxicity testing. ALTEX-Alternatives to Animal Experimentation, 25, 213-231.

Kosachenco, B. G., Lunardi, V. B., Rodrigues, P. R. C., Witz, M. I., Maia, J. Z., Baja, K. G., Rodrigues, N. C. \& Pulz, R. S. 2007. O ensino da cirurgia veterinária com ética e bem-estar animal. Veterinária em Foco, 4, 111-117.

MacPhail, C. M. 2014. Cirurgia dos sistemas reprodutivo e genital. In: Fossum, T. W. (ed.) Cirurgia de pequenos animais. Elsevier, Rio de Janeiro, Brasil.

Quessada, A. M., Sousa, A. A. R., Costa, A. P. R., Adriana, A. S. S. \& Rocha, R. R. C. 2009. Comparação de técnicas de ovariosalpingohisterectomia em cadelas. Acta Scientiae Veterinariae, 37, 253-258.

Read, E. K., Vallevand, A. \& Farrell, R. M. 2016. Evaluation of veterinary student surgical skills preparation for ovariohysterectomy using simulators: a pilot study. Journal of Veterinary Medical Education, 43, 190-213.

Roshier, A. L., Foster, N. \& Jones, M. A. 2011. Veterinary students' usage and perception of video teaching resources. BMC Medical Education, 11, 1-13.

Silva, R. M. G., Matera, J. M. \& Ribeiro, A. A. C. M. 2007. New alternative methods to teach surgical techniques for veterinary medicine students despite the absence of living animals. Is that an academic paradox? Anatomia, Histologia, Embryologia, 36, 220-224.

Silveira, C. P. B., Araújo, M. S. C., Horr, M., Alencar, E. A. M., Rigaud, R., Oriá, A. P.,
Martins Filho, E. F. \& Neto, J. M. C. 2015. Validação de técnica hemostática do complexo arteriovenoso ovariano na ovariosalpingohisterectomia de gatas. Ciência Animal Brasileira, 16, 81-92.

Slatter, D. H. 2007. Manual de cirurgia de pequenos animais. Manole, São Paulo.

Smeak, D. D. 2007. Teaching surgery to the veterinary novice: the Ohio State University experience. Journal of Veterinary Medical Education, 34, 620-627.

Smeak, D. D., N., H. L., L., B. M., A., S. C. \& J., B. S. 1994. Evaluation of an AutotutorialSimulator Program for Instruction of Hollow Organ Closure. Veterinary Surgery, 23, 519528.

Souza, M. C. C. M. I. \& Matera, J. M. 2015. Bleeding simulation in embalmed cadavers: bridging the gap between simulation and live surgery. Altex, 32, 59-63.

Stone, E. A. 2007. Ovário e útero. In: Slatter, R. D. (ed.) Manual de cirurgia e pequenos animais. Manole, São Paulo.

Tefera, M. 2011. Surgical dummy: a surrogate to live animal in teaching veterinary surgery. Ethiopian Veterinary Journal, 15, 1-10.

Tudury, E. A. \& Potier, G. M. A. 2009. Tratado de técnica cirúrgica veterinária. Medvet, São Paulo, Brasil.

Article History:

Received 23 February 2018

Accepted 5 March 2018

Available online 30 April 2018

License information: This is an open-access article distributed under the terms of the Creative Commons Attribution License 4.0, which permits unrestricted use, distribution, and reproduction in any medium, provided the original work is properly cited. 